\title{
CONCENTRACION ATMOSFERICA INVERNAL DE PROPAGULOS FUNGICOS EN UN MERCADO INTE- RIOR DE ABASTOS EN VALPARAISO (CHILE)
}

\author{
(Winter atmosferic concentration of fungal propagules in an indoor \\ food market of Valparaiso (Chile))
}

\author{
M.J. Aira*, E. Piontelli**, \\ V. Jato $* * *$, M. A. Toro.** \\ * Departamento de Botánica, Facultad de Farmacia, \\ Universidad de Santiago,15706 Santiago de Compostela (España). \\ ** Cátedra de Micología, Escuela de Medicina, \\ Universidad de Valparaíso, Casilla 92 V Valparaíso (Chile) \\ *** Departamento de Biología Vegetal y Ciencias del Suelo, \\ Facultad de Ciencias de Ourense, Universidad de Vigo (España)
}

Palabras clave: Propágulos fúngicos, aire, mercado de abastos, Aspergillus, Penicillium, Cladosporium Key words: Fungal propagules, air, food market, Aspergillus, Penicillium, Cladosporium

\section{RESUMEN}

Desde el 17 de abril al 29 de mayo del año 2003, se realizó un estudio de la micota aérea en un Mercado de la ciudad de Valparaíso (Chile), un edificio actualmente destinado al abastecimiento de frutas, verduras y alimentos en general. Mediante un captador de esporas portátil (Burkard Personal Culture), que permite la incorporación de una placa con medio de cultivo (MEA). La periodicidad del muestreo fue semanal, cada vez en tres zonas diferentes en su interior, así como en la atmósfera exterior.

Los resultado muestran una mayor concentra-ción de propágulos fúngicos viables en el aire del interior del edificio (89\%) que afuera (11\%), sobre todo en el primer piso donde la mayoría de los locales se destinan al depósito $y$ venta de frutas y verduras. En dicho lugar, se alcanzan los máximos valores del orden de $6.600 \mathrm{ufc} / \mathrm{m}^{3}$, mientras que en el aire exterior el número máximo de colonias se reduce a la mitad.

En el total de los muestreos se han identificado 27 taxa fúngicos, de los cuales 14, han sido aislados en ambos medios. En el interior, el género más representado fue Aspergillus niger seguido de Penicillium con 12 especies, con la dominancia de P. glabrum, mientras que en el aire exterior Penicillium fue el género más abundante con un total de 9 especies (P. digitatum dominante). Cladosporium cladosporioides, tuvo una representación considerable en ambos ambientes.

\section{ABSTRACT}

Since April 17 to May 19, 1993, the aerial micota of a market in the city of Valparaiso (Chile) was studied, that building being intended at present to supply fruit, vegetables and food, both retail and wholesale. A portable spore detecter (Burkard Personal Culture) which allows to introduce a plate with a culture means (MEA) was used. Periodicity of sampling was weekly, each being carried out in three different zones indoors as well as outdoors.

Results got show a higher concentration of viable fungal propagules in the indoor air (89\%) than outdoors (11\%), especially in the first floor where most shops are designed to store and sell fruit and vegeta-bles. Here, maximum values of colony forming units reach as far as $6,600 u f c / m^{3}$ whereas the maximum number of colonies half decreases outdoors.

Out of the total samplings 27 fungal taxa have been identified, 14 of them being isolated in both media (indoors and outdoors). Inside the market, Aspergillus niger was the genus most represented followed by Penicillium with 12 species identified, P. glabrum being dominant, whereas in the outside air Penicillium was the most abundant genus with a total of 9 species. (P.digitatum dominanting). Cladosporium clados-porioides had a marked representation in both environments. 


\section{INTRODUCCION}

Los hongos son organismos cosmopolitas que pueden desarrollarse en los sustratos más variados (Kustimur \& Yulug, 1977; Moritz \& Martiny, 1997), prácticamente en todos los climas de la tierra e incluso en condiciones extremas o poco estudiadas (Johansen, 1991; Caretta \& Piontelli, 1998; Dose et al. 2001; Tosi et al, 2002). Su ámbito es tan amplio, que sus esporas incluso sobrepasan la atmósfera (Imshenetsky et al., 1976; Viktorov, et al., 1988).

Desde hace décadas, numerosos investigadores se han interesado por el efecto que muchos hongos producen, ya sea en su vertiente clínica, fitopatológica o en el biodeterioro del Patrimonio artístico y monumental (Pitzurra et al., 1999).

El contenido de esporas en la atmósfera se ha estudiado prácticamente en todo el mundo (Barkai-Golan et al., 1977; Larsen, 1981; Abdel-Fattath et al., 1981; Ulutan et al., 1985 ; Palmas et al., 1989; Yang et al., 1989; Shadzi et al., 1993; Takatori et al., 1994; Airaudi \& Marchisio, 1997; Al-Suwaine et al., 1999; Morin, 2001; Pepeljnjak \& Segvic, 2003) y algunos géneros como Alternaria y Cladosporium, han sido objeto de estudios más detallados (Infante et al., 1999, a, b).

Este tipo de investigación, englobada en la rama ambiental de la micología, se ha llevado a cabo tanto en ambientes exteriores como interiores, según fuera el objetivo de la investigación. Algunos trabajos se han centrado en conocer los niveles de propágulos fúngicos en diferentes ambientes de trabajo, con el fin de relacionarlos con determinadas enfermedades ocupa-cionales (Chudnovets, 1998; Levy et al., 1999; Adhikari et al., 2000; Guglielminetti et al., 2001). Otros se dedican al estudio del ambiente interior de las viviendas y centros escolares (Chen \& Chuang, 1975; Oppermann et al., 2001; Etzel, 2001; Chao et al., 2002; Santilli \& Rockwell, 2003; Daisey et al., 2003) o de los centros hospitalarios (Mallea et al., 1983).

La presencia de esporas en el aire exterior se relaciona con los factores climáticos (Burch \& Levetin, 2002; Bustos et al., 2002), lo que permite realizar, en aquellas localidades donde se disponga de una amplia base de datos, modelos predictivos de las concentraciones de esporas en base al clima (Mediavilla et al., 1998).

Son numerosos los trabajos que relacionan la influencia de los hongos en la producción de enfermedades respiratorias de tipo alérgico (Dale et al, 2000; Newson et al, 2000; Singh \& Kumar, 2002). Hardin et al., (2003) señalan que alrededor de un $5 \%$ de los individuos pueden desarrollar alergia a mohos a lo largo de su vida, sin embargo, la intensidad de la respuesta alérgica dependerá de la edad, sexo, mapa genético, estado de salud y de la cantidad y grado de exposición al antígeno (Bardana, 2003). Algunos autores relacionan a determinados géneros con patologías concretas, como es el caso de Penicillium con las infecciones del tracto respiratorio y de Aspergillus con las rinitis (Muller et al., 2002).

En Chile los trabajos de micología ambiental realizados hasta la actualidad, se han centrado casi exclusivamente en la zona central del país. Piontelli \& Velasco (1970), identifican en la atmósfera de Valparaíso una considerable representación de esporas del género Aspergillus y sobre todo de $\boldsymbol{A}$. niger, posteriormente citado en la revisión de flora alergogénica de Chile realizada por Carrasco \& Galleguillos (1973). Estos autores en un trabajo más completo, desarrollado en Valparaíso y Viña del Mar (Piontelli \& Velasco, 1974), identifican por método gravimétrico, 29 géneros en su mayoría hongos filamentosos, destacando la presencia de Cladosporium (43.7\%), Penicillium (19.07\%), Stemphylium (4.3\%), Alternaria (3.6\%), Aspergillus (2 \%) y Rhizopus (1.27\%).

También la capital chilena ha sido estudiada bajo este aspecto. En la parte occidental de la ciudad y también a través de la exposición al aire de placas con medio de cultivo, Urrutia et al., (1976) identifican 16 géneros, cuyos cultivos incuban simultáneamente a $28^{\circ} \mathrm{C}$ y a temperatura ambiente. Entre los hongos filamentosos destacan en orden de importancia en el primer caso Cladosporium, Stemphyllium, Penicillium, Alternaria, Aspergillus y Mucorales, mientras que a temperatura ambiente la secuencia varía de la siguiente manera: Cladosporium, Penicillium, Aspergillus, Alternaria, Stemphyllium y Mucorales.

En esta misma zona de la ciudad Ibáñez et al., (2001), contabilizaron durante un año (1996-1997) las esporas recogidas con un captador Burkard (Burkard Manufacturing Ltd., England). Este instrumento colector se incluye en los sistemas volumétricos no viables. La concentración de esporas osciló entre 308 a 10.334 esporas $/ \mathrm{m}^{3} /$ dia siendo la media anual de 2.154 esporas, aumentando en Abril y Mayo. Identificaron 13 géneros de los cuales los dos más abundantes fueron Cladosporium y Alternaria.

La atmósfera de la zona norte de la capital, también fue objeto de estudio en cuanto a la representación fúngica entre 1991 y 1992 (Ibáñez, 1993; Ibáñez et al., 1998). Dichos autores utilizaron, en este caso, un equipo colector portátil, RCS-Reuter Centrifugal Sampler (RCS Biotest, Frankfurt). Identificaron 39 taxa, con predominancia de los hongos filamentosos, siendo los más abundantes Cladosporium y Ulocladium, ambos especialmente numerosos en verano, mientras Alternaria, Penicillium, fueron más abundantes en invierno y otoño. Aspergillus, fue más abundante en otoño.La concentración de unidades formadoras de colonias a lo largo del período de estudio osciló entre $300 \mathrm{CFU} / \mathrm{m}^{3}$ a $8.850 \mathrm{CFU} / \mathrm{m}^{3}$, siendo la media anual de $1.945 \mathrm{CFU} / \mathrm{m}^{3}$. No encontraron diferencias en los resultados desde el punto de vista espacial en las diez zonas 
período de estudio osciló entre $300 \mathrm{CFU} / \mathrm{m}^{3}$ a $8.850 \mathrm{CFU} /$ $\mathrm{m}^{3}$, siendo la media anual de $1.945 \mathrm{CFU} / \mathrm{m}^{3}$. No encontraron diferencias en los resultados desde el punto de vista espacial en las diez zonas muestreadas en la ciudad de Santiago, pero se detectaron variaciones estacionales, con aumento de la representación fúngica en verano desde Septiembre a Abril, cuando las temperaturas son más elevadas y la humedad menor, disminuyendo de Junio a Agosto cuando las temperaturas son menores y aumenta la humedad.

Por lo que se ha comentado anteriormente, la información aeromicológica en Chile sigue siendo en la actualidad escasa y limitada. Son necesarios sobre todo estudios en ambientes de interior, donde las peculiaridades del lugar puedan favorecer el desarrollo de hongos, cuyas esporas supongan un posible riesgo a la salud (Thompson et al ., 1994).

Por ello, el objetivo de este trabajo ha sido analizar el contenido de esporas en el ambiente interior de un mercado, donde era de esperar concentraciones elevadas de propágulos fúngicos debido a la naturaleza de los productos en venta y depósito de dicho lugar.

\section{MATERIAL Y MÉTODOS}

El presente estudio se llevó a cabo en el Mercado Cardonal, uno de los edificios emblemáticos de Valparaíso de gran valor arquitectónico, que actualmente es utilizado como Mercado de abastos. En este lugar muy concurrido, se efectúa una buena parte del abastecimiento mayorista de la ciudad en cuanto a frutas, verduras y alimentos en general. Fue construido en 1907, tiene dos pisos y unos 200 locales. Su arquitectura es muy peculiar ya que consta de un armazón de vigas de hierro y acero a la vista.

La toma de muestras se realizó con un captador volumétrico portátil (Burkard Personal Culture), que permite la incorporación de una placa con medio de cultivo, en nuestro caso agar extracto de malta (MEA). Se realizaron siete muestreos, todos los jueves por la mañana desde el 17 de abril hasta el 29 de mayo del año 2003 en tres puntos del interior del Mercado y en el exterior del mismo. La muestra 1 (que llamaremos $\mathrm{Ml}$ ), corresponde al primer piso donde la mayoría de los locales se dedican a la venta de frutas y verduras, se eligió una localización apartada de la puerta de entrada del edificio, para evitar en lo posible, la mezcla con el aire exterior. Las muestras 2 y 3 (M2 y M3) se tomaron en el segundo piso del edificio; M2 abarca la zona de carnicería y venta de aves vivas y la M3, la zona de comedores. Por su parte la muestra 4 (M4) se tomó en el exterior del edificio, donde también hay numerosos puestos ambulantes de pescado, verduras y flores. En todos los casos el captador se mantuvo a una altura aproximada de 1 metro del suelo y el tiempo de muestreo fue de dos minutos por muestra, lo que hace que los resultados obtenidos puedan ser comparados.

Una vez recogidas las muestras, las diferentes placas se incubaron en estufa a $25^{\circ} \mathrm{C}$ durante 7 dias, aunque se realizó un control a partir de los dos días de muestreo con el fin de contabilizar las colonias de hongos de rápido crecimiento como es el caso de los Mucorales. Con esta excepción, el recuento de número de colonias se realizó en la parte posterior de la placa, a la semana de muestreo. Para el cálculo de unidades formadores de colonias (ufc) por $\mathrm{m}^{3}$ de aire, se multiplicó el número de colonias de cada placa (corregido por tabla estándar) por mil, y dicho valor, se dividió por el producto de multiplicar el flujo de aire del captador (10 $1 /$ minuto) por el tiempo de muestreo ( 2 minutos).

Además en cada uno de los muestreos se tomaron los datos correspondientes a temperatura y humedad con una estación meteorológica portátil (Tabla 1), con el fin de comparar los resultados obtenidos en cuanto al número de colonias fúngicas viables con la climatología, utilizando el programa informático Statistica. No se han tenido en cuenta las precipitaciones ya que han estado ausentes durante todo el período de muestreo.

\section{RESULTADOS}

\section{1.- Análisis cuantitativo}

Los resultados obtenidos indican una mayor concentración de propágulos fúngicos en el aire del interior

Tabla 1.- Datos de Temperatura $\left({ }^{\circ} \mathrm{C}\right)$ y Humedad (Hdd \%)

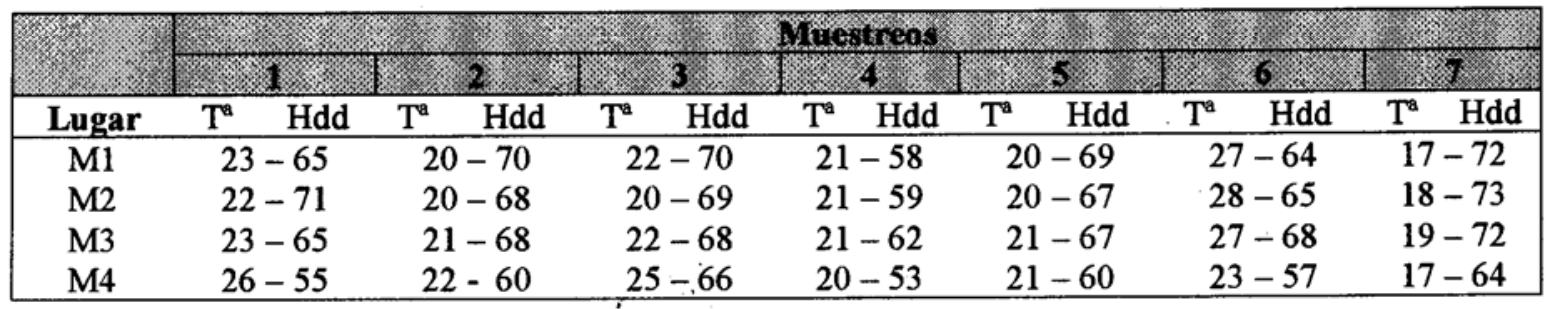


centración de propágulos fúngicos en el aire del interior del Mercado, donde se contabilizaron $12.578 \mathrm{ufc} / \mathrm{m} 3$ $(88.7 \%)$ como valor promedio en los siete muestreos realizados, frente a las $1.606 \mathrm{ufc} / \mathrm{m} 3(11.7 \%) \mathrm{del}$ aire exterior (Fig. 1).

En cuanto a la distribución en las distintas zonas de muestreo, los valores obtenidos en el primer piso (M1) fueron los más elevados con $5.233 \mathrm{ufc} / \mathrm{m}^{3}$ de promedio en los siete muestreos realizados, que representan un $37 \%$ del total de las colonias contabilizadas. Por su parte en el segundo piso (M2, M3), los niveles registrados han sido similares ( 3.377 y $3.968 \mathrm{ufc} / \mathrm{m}^{3}$ respectivamente) lo que representan un $24 \%$ y $28 \%$ del total. Los valores del aire exterior (M4) han sido mucho más bajos, con un total de $1.606 \mathrm{ufc} / \mathrm{m}^{3}$ contabilizadas, lo que supone un $11 \% \mathrm{del}$ total.

Analizando con más detalle los resultados desde el punto de vista cuantitativo, se observó que en la primera zona de muestreo (M1) los valores oscilaron entre 2.280-6650 ufc/ $/ \mathrm{m}^{3}$, alcanzándose el valor máximo el día 29 de mayo y una cantidad próxima la semana anterior $\left(6.450 \mathrm{ufc} / \mathrm{m}^{3}\right)$. En la segunda zona de muestreo (M2), se obtuvieron valores similares, oscilando en este caso entre 2.280-6600 ufc $/ \mathrm{m}^{3}$ y alcanzándose el valor máximo, también en el último muestreo.

En la tercera zona de muestreo (M3), el número de unidades formadoras de colonias osciló entre 2.280$5700 / \mathrm{m}^{3}$ y el máximo se logró el día l de mayo. Excluímos de valoración el dato obtenido el 15 de mayo ya que no se ha observado ninguna razón que permita justificar dicho resultado, por tanto es considerado como anómalo. Finalmente, en la atmósfera externa (M4), las concentraciones fúngicas oscilaron entre $900 \mathrm{ufc} / \mathrm{m}^{3}$ y poco más de $3.000 \mathrm{ufc} / \mathrm{m}^{3}$, alcanzándose el valor máximo, también el primer día del mes de mayo.

\section{2.- Relación con los parámetros meteorológicos}

La correlación entre el número de $\mathrm{ufc} / \mathrm{m}^{3}$ de aire

Fig. 1.- Porcentaje de ufc/m3 en los cuatro puntos de muestreo

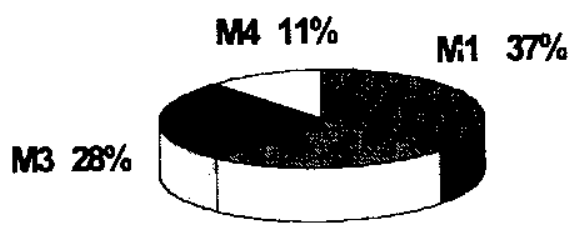

M2 $24 \%$

$\mathrm{Ml}=$ primer piso;M2, M3= segundo piso;M4= Exterior
Tabla 2.- Análisis de correlación

\begin{tabular}{|llllll}
\hline Temp. & -.145455 & -.185312 & +.261785 & +.414431 \\
TR & +.154545 & -.107143 & +.523570 & +.454545 \\
\hline
\end{tabular}

en cada muestreo y los parámetros meteorológicos, ha tenido un indice de significación inferior al $90 \%$ en todos los casos, probablemente, debido a que las variaciones de temperatura y humedad a lo largo del período de estudio han sido escasas y el número total de datos obtenidos relativamente bajo para obtener una alta significación desde el punto de vista estadístico (Tabla 2). En la mayoría de los casos, la humedad ha resultado positiva, destacando la influencia de dicho factor en M3, donde permite explicar hasta un $52 \%$ de los datos obtenidos.

\section{3.- Análisis cualitativo}

En los muestreos realizados tanto en el interior como en el exterior del Mercado, se han identificado un total de 27 taxa fúngicos diferentes, de los cuales 14 son comunes en ambos medios (Tabla 3 ), mientras que Aspergillus ochraceus, Penicillium expansum, $P$. purpurogenum, P.simplicissimum y $P$. viridicatum solo se han identificado en el interior del mercado y Acremonium sp., Alternaria alternata complex, Aspergillus sydowii, Aureobasidium pullulans var. melanigenum, Penicillium fellutanum, P. janczewskii, P. janthinellum y Phoma spp. solo aparecieron formando parte de la micota fúngica del aire exterior.

La diversidad fúngica, en el total de los 7 muestreos realizados, ha sido ligeramente inferior en la atmósfera interna del Mercado con respecto al exterior, con una relación de 19 a 22 taxa identificados respectivamente. Analizando el número de taxa diferentes en cada uno de los muestreos (Tabla 4), se observa que los valores totales y medios son similares tanto en el interior del mercado como afuera, del orden de 7-7 taxa en promedio. La mayor diversidad en el interior del mercado se registró el 24 de abril con 25 taxa en los tres puntos de muestreo mientras que en el exterior se produjo el día 15 de mayo con 10 taxa identificados.

\section{4.- Abundancia y frecuencia de aislamientos}

En el total de muestras recogidas en el interior del mercado se han realizado 1.904 aislamientos de los cuales se han identificado un $84 \%$, resultando el resto micelios estériles que no llegaron a esporular (Tabla 5). La especie más abundante ha sido Aspergillus niger con un $45.8 \%$ de representación con respecto al total, seguido del género Penicillium que alcanzó un 31.7\%.; ambos 
Tabla 3.- Hongos detectados en el aire interior $y$ exterior

\begin{tabular}{l} 
TAXA COMUNES \\
\hline Aspergillus alliaceus Thom \& Shurch \\
Aspergillus niger van Tieghem \\
Cladosporium cladosporioides (fres) de Vries \\
Mucor plumbeus Bonord \\
Paecilomyces variotil Bain \\
Penicillium aurantiogriseum complex Dierckx \\
Penicillium brevicompactum Dierckx \\
Penicillium chrysogenum Thom \\
Penicillium decumbens Thom \\
Penicillium digitatum Sacc. \\
Penicillium glabrum (Wehmer) Westling \\
Penicillium thomii Maire \\
Rhizopus oryzae Went \& Prinsen Geerlings \\
Rhodotorula sp.
\end{tabular}

taxa presentan una frecuencia del $100 \%$ en los muestreos. Con menor importancia se presentan Cladosporium cladosporioides (3.9\%) y diversos Mucorales (2.8\%).

Por su parte en la atmósfera externa se han aislado 282 colonias fúngicas, de las cuales se ha identificado un $78 \%$, resultando las demás estériles. En ellas el género predominante fue Penicillium, que alcanzó un $30 \%$ de representación seguido de Cladosporium cladosporioides con un $27.3 \%$ y con valores inferiores, A. niger (15,2\%) y Mucorales (3.9\%).

La diversidad fúngica dentro del género Aspergillus ha sido baja ya que sólo se han identificado tres especies en el interior del mercado y otras tantas en la atmósfera exterior. Dos de ellas, Aspergillus niger y A. alliaceus, han resultado comunes en ambos medios mientras que A. ochraceus solo ha estado representada en el aire del mercado y A.sydowii en el exterior.

Otro de los géneros con una considerable significación, ha sido Cladosporium cladosporioides, concretamente en la atmósfera externa donde se identificó en la totalidad de los muestreos realizados. En cuanto a los Mucorales, se han identificado Rhizopus oryzae (más abundante en el interior del mercado) y Mucor plumbeus, que por el contrario, se encontró con mayor frecuencia en el aire exterior.

Haciendo un análisis más detallado de los resultados obtenidos con el género Penicillium (Tabla 6), se observa que en la atmósfera del interior del mercado se han identificado un total de 12 especies diferentes, de las cuales las más abundantes han sido $\boldsymbol{P}$. glabrum $(22.9 \%)$, $\boldsymbol{P}$. decumbens (20\%), $\boldsymbol{P}$. brevicompactum $(16.7 \%)$ y $\boldsymbol{P}$. simplicissimum (14.9\%). En cuanto a su frecuencia $\boldsymbol{P}$. brevicompactum es la única especie que aparece en el $100 \%$ de los muestreos realizados y $\boldsymbol{P}$. glabrum en el
Tabla 4.- Diversidad fúngica

\begin{tabular}{|lccccccccc|}
\hline \multicolumn{10}{|c|}{ Muestreos } \\
\hline & $\mathbf{1}$ & $\mathbf{2}$ & $\mathbf{3}$ & $\mathbf{4}$ & $\mathbf{5}$ & $\mathbf{6}$ & $\mathbf{7}$ & Tot. & Media \\
\hline M1 & 4 & 7 & 6 & 6 & 9 & 7 & $\mathbf{3}$ & 42 & 6 \\
M2 & 4 & 11 & 8 & 3 & 9 & 6 & 7 & 48 & 7 \\
M3 & 4 & 7 & 4 & 7 & 4 & 11 & 6 & 43 & 6 \\
Total & 12 & 25 & 18 & 16 & 22 & 23 & 16 & & \\
\hline M4 & 7 & 7 & 5 & 5 & 10 & 8 & 6 & 48 & 7 \\
\hline
\end{tabular}

$86 \%$ de los mismos.

Por su parte en el aire externo se han identificado 9 especies diferentes, siendo $\boldsymbol{P}$. digitatum la más abundante $(32.4 \%)$ seguida de $\boldsymbol{P}$. brevicompactum y $\boldsymbol{P}$. decumbens, ambas con la misma representación (16.2\%) además de $\boldsymbol{P}$. glabrum (10.8\%). La especie más frecuente en los muestreos (43\%) fue P. brevicompactum.

\section{DISCUSION}

Uno de los problemas en los estudios de micología ambiental, estriba en que los resultados varían en función del método de muestreo utilizado (gravimétrico o volumétrico), del sistema colector en el caso de la captación volumétrica (Bellin \& Schillinger, 2001) y de las propias características del lugar de muestreo. Pero quizás, el factor que más condiciona es, si el muestreo se realiza en la atmósfera exterior o en un ambiente de interior, en cuyo caso la representación mayoritaria de los propágulos fúngicos en las muestras, corresponderá a la micota local.

En Chile, la mayoría trabajos realizados hasta el momento se han centrado en el exterior en la zona central y no han seguido una metodología homogénea, por ello, los valores de representación para los diferentes taxa son variables. Así, Cladosporium se cita con valores que oscilan entre $29 \%-70,9 \%$, Penicillium entre $5,7 \%$ a $19,07 \%$, Alternaria entre 1,9\%-6\% y Aspergillus entre 2-7,2\% (Piontelli \& Velasco, 1974; Urrutia et al., 1976; Ibáñez et al., 1998, 2001). Estos resultados no difieren mayormente de los de otras ciudades latinoamericanas

Tabla 5.- Número total de aislamientos y frecuencia de los hongos más abundantes en los muestreos realizados

\begin{tabular}{|l|c|c|c|c|}
\cline { 2 - 5 } \multicolumn{1}{c|}{} & \multicolumn{2}{c|}{ INTERIOR } & \multicolumn{2}{c|}{ EXTERIOR } \\
\cline { 2 - 5 } \multicolumn{1}{c|}{} & Totales & Frec & Totales & Frec \\
\hline A. niger & 873 & $100 \%$ & 43 & $71 \%$ \\
Otros Aspergillus & 3 & $28 \%$ & 4 & $28 \%$ \\
Penticilium spp. & 604 & $100 \%$ & 85 & $100 \%$ \\
C. cladosporioides & 75 & $57 \%$ & 7 & $100 \%$ \\
Mucorales & 54 & $86 \%$ & 11 & $57 \%$ \\
\hline
\end{tabular}


ción, especialmente en las naranjas (Domsch et al.,1980). Mucha de la fruta se exhibe en la zona externa del mercado, de allí su posible relación con la mayor abundancia. A pesar que, la humedad relativa ha sido siempre mayor en el interior del mercado, situación que favorecería su germinación en este ambiente (necesita mayor actividad de agua mayor $(0,90)$ que P.brevicompactum), la dominancia de $\boldsymbol{A}$. niger, un hongo de más rápido crecimiento que P.digitatum pueden ser una de las causas de su menor presencia.

Una de las dificultades a la hora de interpretar los resultados obtenidos, es la escasez de referencias bibliográficas en cuanto a los niveles (de esporas o, en nuestro caso, de unidades formadoras de colonias), que pueden considerarse nocivos para la salud humana. Klanova (2000), señala que en ambientes interiores la concentración de propágulos fúngicos superior a $2.000 \mathrm{CFU} / \mathrm{m}^{3}$, puede considerarse un factor de riesgo. En este sentido debe resaltarse que los valores promedio de los siete muestreos realizados en el mercado superan dichas cifras, independientemente del punto de muestreo, ya que oscilaron entre $3.377 \mathrm{CFU} / \mathrm{m}^{3}$ y $5.233 \mathrm{ufc} / \mathrm{m}^{3}$ (valores promedio), mientras que en el aire exterior se reducen a $1.606 \mathrm{ufc} / \mathrm{m}^{3}$.

Sin embargo, el daño que estos hongos puedan causar a la población depende en parte del poder patógeno del hongo (Fischer \& Dott, 2003) y del tiempo de exposición al antígeno. En cuanto a la respuesta alérgica de la población chilena a las esporas de los hongos, cabe resaltar que, en pruebas cutáneas, el género que da valores más elevados es Alternaria (11.8\%) seguido de Aspergillus
(7.5\%), Penicillium (6.5\%) y Cladosporium (5.1\%) según los datos aportados por Ibáñez et al., (2001). Por su parte Mallol et al., (2000), señalan que en las últimas décadas, la prevalencia de asma en niños de edad escolar ha ido en aumento, siendo incluso superior en Chile que en otros países (Robertson et al., 1993).

Independientemente de estos datos, a futuro será necesario revalorar los datos obtenidos frente a medidas de limpieza y ventilación acordes a este ambiente, así como un control más profundo de los productos a la venta y depósito, los que contribuirían a disminuir las elevadas concentraciones de propágulos fúngicos en el aire.

\section{REFERENCIAS}

Abdel-Fattath, H.M.; Mousbasher, A.H. \& Swelim, M.A. (1981). Studies on airborne fungi at Qena.II. Diurnal fluctuations. Z. Alleg Mikrobiol. 21:177-179

Adhikari, A.; Sen, M.M.; Gupta, S. \& Chanda, S. (2000). Incidence of allergenical significant fungal aerosol in a rural bakery of West Bengal, India. Mycopathologia 149:35-45

Airaudi, D. \& Marchisio, V.F. (1997). Fungal biodiversity in the air of Turin. Mycopathologia 136:95-102

Al-Suwaine, A.S.; Bahkali, A.H. \& Hasnain, S.M. (1999). Seasonal incidence of airborne fungal allergens in Ryadh, Saudi Arabia. Mycopathologia 145:15-22

Bardana, E.J. (2003). Indoor air quality and health does fungal contamination play a significant role?. Immunol Allergy Clin North Am 23: 291-309

Barkai-Golan, R.; Frank, M.; Kantor, D.; Karadavid, R. \& Toshner, D. (1977). Atmospheric fungi in the desert town of Arad and in the coast plain of Israel. Ann. Allergy 38:270-274

Bellin, P. \& Schillinger, J. (2001). Comparison of field performance of the Andersen N6 single stage and the SAS sampler for airborne fungal

propagules. Indoor Air 11: 65-68

Burch, M. \& Levetin, E. (2002). Effects of meteorological conditions on spore plumes. Int J Biometeorol 46:107-117

Bustos, I.; Angulo, J. \& Domínguez, E. (2002). Caracterización aeromicológica de la atmósfera del Parque Natural de la Sierra de Hornachuelos (Córdoba), relación con los parámetros meteorológicos. Libro de textos completos del XIII Simposio de la APLE, Cartagena, España, pp.61-66

Caretta, G. \& Piontelli, E. (1998). Preserved ascomatal and other fungal structures on the remains of a ninth century Longobard abbess exhumed from a Monastery in Pavia, Italia. Mycopathologia 140:77-83

Carrasco, F. \& Galleguillos, F. (1973). Características geografico-climáticas y flora alergogénica de Chile. Rev Méd Chile 101:155-162

Chao, H.J.; Milton, D.K.; Schwartz, J. \& Burge, H.A. (2002). Dustborne fungi in large office buildings. Mycopathologia 154: 93-106

Chen, C.Y. \& Chuang, C.Y. (1975). Fungi isolated from asthmatic homes in the Taipei area. Zhonghua Min Guo Wei Sheng Wu Xue Za Zhi, 8:253-258
Chudnovets, AIA (1998). Microflora as a risk factor for workers in the manufacture mixed feeds. Lik Sprava 7:170-174

Daisey, J.M.; Angell, W.J. \& Apte, M.G. (2003). Indoor air quality, ventilation and health symptoms in schools an analysis of existing information. Indoor Air 13:53-64

Dale, R.E.; Cakmak, S.; Burnett, R.T.; Judek, S.; Coates, F. \& Brook, J.R. (2000). Influence of ambient fungal spores on emergency visits for asthma to a regional children's Hospital. Am. J. Respir. Crit. Care. Med. 162:2087-2090

Domsch, K.H.; Gams, W. \&Anderson T-H. (1980). Compendium of soil fungi Vol.1. Academic Press.London, N.Y.

Dose, K.; Bieger-Dose, A.; Ernst, B.; Feister, U.; Gómez-Silva, B.; Klein, A.; Risi, S. \& Stridde, C. (2001). Survival of microorganisms under the extreme conditions of Atacama Desert. Orig. Life Evol. Biosph. 31:287-303

Etzel, R.A. (2001). Indoor air pollutants in homes and schools. Pediatr. Clin. North. Am. 48:1153-1165 
Fischer, G. \& Dott, W. (2003). Relevance of airborne fungi and their secondary metabolites environmetal occupational and indoor hygiene. Arch. Microbiol. 179:75-82

Guglielminetti, M.; Valoti, E.; Cassini, P.; Taino, G. \& Caretta, G. (2001). Respiratory syndrome very similar to extrinsic allergic alveolitis due to Penicillium verrucosum in workers in a cheese factor. Mycopathologia 149: 123-129

Hardin, B.D.; Kelman, BJ. \& Saxon, A. (2003). Adverse human health effects associated with moulds in the indoor environment. J. Occup. Environ. Med. 45: 470-478

Ibáñez, V. (1993). Fluctuación estacional de bioalergenos: estudio cuantitativo y cualitativo de hongos anemófilos en Santiago norte 1991-1992, con especial referencia al género Aspergillus. Tesis de Magíster. Facultad de Medicina. Universidad de Chile

Ibañez, V.; Rojas, G. \& Roure, J.M. (2001). Airborne fungi monitoring in Santiago, Chile. Aerobiologia 17:137-142

Ibáñez, V.; Thompson, L. \& Mañalich, J. (1998). Fluctuación estacional de hongos anemófilos en Santiago Norte-Chile. Boletín Micológico $13: 47-56$

Imshenetsky, A.A.; Lysenko, S.V.; Kazakov, G.A. \& Ramkova, N.V. (1976). On microorganism of the stratosphere. Life Sci. Space Res. 14: 359-362

Infante, F.; Alba, F.; Caño, M.; Castro, A.; Domínguez, E.; Méndez, J.; \& Vega, A. (1999a). A comparative study of the incidence of Alternaria conidia in the atmosphere of five spanish cities. Polen 10:7-15

Infante, F.; Castro, A.; Domínguez, E.; Guardia, A.; Méndez, J.; Sabariego, S. \& Vega, A. (1999b). A comparative study of the incidence of Cladosporium conidia in the atmosphere of five spanish cities. Polen 10: $17-25$

Javeed, I.H. ; Zaid, I.H. \& Anees, S. (1994). Incidence of fungal flora on some vegetable market at Hyderabad (A.P.). $5^{\text {th }}$ International Conference on Aerobiology, Bangalore, India, 65.

Johansen, S. (1991). Airborne pollen and spores on the Arctic island of Jan Mayen. Grana 30:373-379

Kakde, U.B.; Kakde, H.U. \& Saoji, A.A. (2001). Seasonal variation of fungal propagules in a fruit market environment, Nagpur (India). Aerobiologia 17:177-82

Klanova, K. (2000). The concentrations of mixed populations of fungi indoor at rooms with and without mould problems; rooms with and without health complaints. Cent. Eur. J. Public Health 8:59-61

Kumar, R. \& Gautam, P.L. (1994). Aeroallergenic significance of interactions between pollen, fungi and mites. $5^{\text {th }}$ International Conference on Aerobiology, Bangalore, India, 135

Kustimur, S. \& Yulug, N. (1977). The mycological and bacteriological flora of cigarettes and relation to human throat culture and inhalation air. Mikrobiol. Bul. 11:45-60

Larsen, L.S. (1981). A three-year-survey of microfungi in the air of Copenhagen 1977-79. Allergy 36:15-22
Levy, J.I.; Nishioka, Y.; Gilbert, K.; Cheng, C.H. \& Burge, H.A. (1999). Variabilities in aerosolizing activities and airborne fungal concentrations in a bakery. Am. Ind. Hyg. Assoc. J. 60:317-325

López, R.; Ruiz, D.; Guadalupe, J. \& Esquenaz, M.T. (1986). Seasonal variation of allergy-causing fungi in the southern of México City. Allergol. Immunopathol. 14:43-8

Mallea, M.; Renard, M. \& Charpin, J. (1983). Fungal flora in a hospital environment. Sem. Hosp. 59:2113-2117

Mallol, J.; Cortez, E.; Amarales, L.; Sanchez, I.; Clavo, M. ; Soto, S. ; Strickle, A.; Kyling, A. ; Sanhueza, I. \& Albornoz, C. (2000). Prevalence of asthma in Chilean students. Descriptive study 24,470 children. ISAAC-Chile. Rev. Med. Chil. 128:279-285

Mediavilla, A.; Angulo, J.; Infante, F.; Comtois, P. \& Domínguez, E. (1998). Preliminary stadistical modeling of the presence of two conidial types of Cladosporium in the atmosphere of Córdoba, Spain. Aerobiologia 14:229- 234

Morin, O. (2001). Airborne moulds in Nantes. Effect of climatic factors. Allerg. Immunol. 33:100-101

Moritz, M. \& Martiny, H. (1997). Fungi and bacteria on air filters from heating, ventilation a air-conditioning systems: a method for determination of fungal and bacteria on air filters. Zentralb. Hyg. Umweltmed. 199: $513-526$

Muller, A.; Lehmann, I.; Seiffart, A. ; Diez, U. ; Wetzig, H. ; Borte, M. \& Herbarth, O. (2002). Increased incidence of allergic sensitisation and respiratory diseases due to mould exposure: results of the Leipzig Allergy Risk Study (LARS). Int. J. Hyg. Environ. Health 204:363-365

Newson, R.; Strachan, D.; Corden, J. \& Millington, W. (2000). Fungal and other spore counts as predictors of admissions for asthma in the Trent region. Occup. Environ. Med. 57:786-792

Oppermann, H.; Doering, C.; Sobottka, A.; Kramer, U. \& Thriene, B. (2001). Exposure status of East and West German households with house dust mites and fungi. Gesundheitswesen 63:85-89

Palmas, F.; Murgia, R.; Deplano, N.; Fadda, ME \& Cosentino, S. (1989). Results of an airborne spore study in various regions of southern Sardinia. Ann. Ig. 1:1647-1656

Pepeljnjak, S. \& Segvic, M. (2003). Occurence of fungi in air and on plants in vegetation of different climatic regions in Croatia. Aerobiologia 19:11-19

Piontelli, E. \& Velasco, M.O. (1970). El género Aspergillus y la flora micótica del aire de Valparaíso, Chile. X Congreso Internacional de Microbiología, México, 1970.

Piontelli, E. \& Velasco, M.O. (1974). Flora anemófila en Valparaíso, Chile. Rev. Med. Chile 102:104-108

Pitzurra, L.; Belleza, T.; Giammarioli, M.; Giraldi, M.; Sbaraglia, G.; Spera, G. \& Bistoni, F. (1999). Microbial environmental monitoring of the refectory in the monastery of St. Anna in Foligno. Italy. Aerobiologia 15:203-209

Pugalmaran, M. \& Vittal, B.P.R. (1994). Airborne moulds in leather storage and their role in biodeterioration of leather. $5^{\text {th }}$ International Conference on Aerobiology, Bangalore, India, 74

Robertson, C.F.; Bishop, J.; Sennhauser, F.H. \& Mallol, J. (1993). 
International comparison of asthma prevalence in children: Australia, Switzerland, Chile. Pediatr. Pulmonol. 16:219-226

Rosas, I.; Escamilla, B.; Calderón, C. \& Mosiño, P. (1990). The daily variations of airborne fungal spores in México City. Aerobiologia 6:153-158

Samson, R.A.; Hoekstra,E.S.; Frisvald, J.C. \& Filtenborg,O. (2000). Introduction to food-and airborne fungi, Sixth Edition CBS.Utrecht.

Santilli, J. \& Rockwell, W. (2003). Fungal contamination of elementary schools: a new environmental hazard. Ann. Allergy Asthma Immunol. 90(2):203-208

Senkpiel, K. Kurowski, V. \& Ohgke, H. (1996). Indoor air studies of mould fungus contamination of homes selected patients with bronchial asthma (with special regard evaluation problems. Zentralbl. Hyg. Umweltmed. 198:191-203

Shadzi, S.; Zahraee, M.H. \& Chadeganipour, M. (1993). Incidence of airborne fungi in Isfahan, Iran. Mycoses 36: 69-73

Simsekli, Y.; Gücin, F. \& Asan, A. (1999). Isolation and identification of indoor airborne fungal contaminants of food production facilities and warehouses in Bursa, Trukey. Aerobiologia 15:225-231

Singh, A. \& Singh, A.B. (1999). Aspergillus spp. As an important occupational risk factor among susceptible individuals. Aerobiologia 15:223-240

Singh, A.B. \& Kumar, P. (2002). Common environmental allergens causing respiratory allergy. India. Indian J. Pediatr 69:245-250

Takatori, M.; Shida, T.; Akiyama, K. \& Takatori, K. (1994). Airborne fungi during the last ten years in Sagamihara. Arerugi 43:1-8

Thompson, L.; Castrillón, M.A.; Delgado, M. \& García, M. (1994). Aislamiento del género Aspergillus de la tierra de plantas ornamentales intrahospitalarias. Rev. Med. Chile 122:1367-1371

Tosi, S.; Casado, B.; Gerdol, R. \& Caretta, G. (2002). Fungi isolated from Antartic mosses. Polar Biol. 25:262-268

Trujillo, D.; Infante, F.; Galán, C \& Domínguez, E. (1990). Seasonal and daily variation of Aspergillus Mich. ex Fr. spores in the atmosphere of Córdoba (Spain). Allergol. et Immunopathol. 18:167-173

Ulutan, F.; Copur, S. \& Kocoglu, T. (1985). The fungal flora of the air in the villages around Carsamba. Northern Turkey. Mikrobiol. Bul. 19:139-143

Urrutia, J.; Orellana, M; Muñoz, J.M.; Carras, E. \& Troncoso, A. (1976). Estudio micológico en el área occidente de Santiago. Rev. Med. Chile 104:213-215

Viktorov, A.N.; Novikova, N.D.; Deshevaia, E.A.; Polikarpov, N.A; Poddub, S.V. \& Bragina, M.P. (1988). Comparative evaluation of microorganisms biological characteristics isolated in the orbital complex "MIR" on differents phases of its operation. Aviakosm Ekolog. Med. 32(2):61-68

Yang, J.P.; Yin, Q.Z.; Ye, M.J.; Zhang, C.W. \& Yu, Q.A. (1989). Quantitative study of airborne fungi at three functional section of Chengdu city. Hua Xi Yi Ke Da Xue Bao 20:448-451 